\title{
Research on Speed-Up Method of SURF Through Properties Analysis
}

\author{
She Xuebing \\ Jiangxi Institute of Technology, Nanchang, China \\ shexuebing1982@sohu.com
}

Keywords: SURF; Properties analysis; Speedup; Resesrch on

\begin{abstract}
To resolve the problem of a little bit low speed the typical image feature extraction algorithm SURF ( speeded up robust feature ) has, in the paper we do an in-depth analysis on the algorithm properties in regard to its running time, instruction type, memory access, hot spots, and etc. On this basis,we optimize the SURF algorithm by reconstructing the judgment logic,the memory accessing and data organisations, the library functions implementation and other strategies.
\end{abstract}

\section{Introduction}

With the diversification of information needs and comprehensive application of multimedia data, the center has become the main type of Internet application. Image retrieval algorithm as the basic algorithm of multimedia information retrieval, is widely used in such applications. Current image matching algorithm is mainly divided into based on global features and local characteristics of the two kinds of. Based on all the feature algorithm uses image some features to describe an image, a small quantity of information, fast processing speed, but the accuracy and robustness is limited. Local feature algorithms use hundreds of features to describe a picture or video , with high accuracy, and in the image of the various changes can still achieve high retrieval effectiveness, therefore become a hot research and application at home and abroad to come this year[1].

SURF is a local image features extraction algorithm based on SIFT, by virtue of its excellent performance, has gradually become the mainstream algorithm to extract the image layout characteristics, However, since the SURF algorithm requires the feature point location and description of the complex, the processing speed is very slow, difficult to meet the real time requirement of many systems: In the present mainstream processor architecture, SURF can only finish 3 pictures per second[2].

\section{Feature extraction algorithm of SURF}

SURF is a new SIFT image extraction algorithm based on the local characteristic, mainly includes three steps: description algorithm initialization, feature detection and feature.[3]

Read the image, to initialize variables. Then through the OpenCV Library of the original image into gray image. Finally, to reduce duplication of memory access and calculated by the gray image is converted to integral image, in order to improve the performance of feature detection and signs described[4].

This stage is used to detect the input of all feature points in the image. The first generation of Pyramid Gauss Gauss by using convolution and images, using Gauss differential and convolution Gauss image difference in Pyramid, and then construct the scale space extreme points in the scale space, and then calculated within $3 \times 3 \times 3$ stereo field, as the feature point. In the concrete implementation, using Hessian matrix, SURF box filter and integral image to simplify the operation[5].

Description: the stage feature vector is used to describe each selected feature points, for the subsequent matching processing to provide enough information. The first step is to calculate the direction of each feature vector, the principal direction. The second step is to select features region size is 20 scale $\mathrm{x} 20$ scale in the main direction (scale feature detection using scale), which can be 
divided into 4 sub regions in X 9 x $9=814$ sampling points of Haar wavelet transform. Each sub region with the vector ( $\sum d x, \sum d y, \sum|\mathrm{dx}|, \sum|\mathrm{dy}|$ ) expressed vector SURF, so feature described as $4 \times 4 \times 4=64$ dimension.

\section{Optimization of SURF algorithm and Implementation}

Based on the analysis results as a guide, we on the existing SURF algorithm is optimized from the aspects of reducing branch instructions, simplify the library function and removing the redundant memory access[6]. The main optimization strategies used are: reconfigurable logic, reconstruction of library functions, reconstruction of memory access. Optimization strategy of all to ensure the accuracy of the premise of algorithm $100 \%$.

In order to reduce the performance overhead caused a lot of branch instructions, we use "read extended array" judgment method instead of the original BoxIntegral function, greatly improves the performance of the function. In order to realize the strategy, first of all need the array proved offset calibration position to read an extended boundary to replace the original boundary detection.

Analysis from the front, a lot of statements to determine conditions for the existence of BoxIntegral function, its function is to detect array bounds, prevent read integral image for array bounds error. Details of the process are as follows: first, the high detection detection of cross-border: compared with the existing maximum and wide, high, low and detection; position: high cross-border and slightly different, here if cross-border directly returns 0 , read the integral image and forces coordinate transform.[7]

The BoxIntegral parameter has four, are to be rectangular gray and the upper left corner of the $\mathrm{X}$ coordinates, Y coordinates, rectangular, rectangular. The BoxIntegral parameter has a total of four, are to be rectangular gray and the upper left corner of the $\mathrm{X}$ coordinates, $\mathrm{Y}$ coordinates, rectangular, rectangular. Therefore, only need to analysis BoxIntegral the caller of the function, scope and the incoming parameters, can deduce the cross range.

BuildDet function calls, to calculate characteristics of Pyramid transform on each scale all point values. Here the need to calculate the point is a pixel on the original image, and the integral image and binary image the same wide and high, so this case cannot happen out of.

By the getOrientation function call, to calculate the characteristic direction of each feature point. The current feature point as the center of computing point to here, (6 X scale) as the radius of all round, not including the circumference. Occurrence of cross-border may in this case, and the range of $(0.5 \mathrm{X}$ scale). Scope definition may be derived by SURF algorithm for scale $(1,12)$. Therefore in this case the array bounds range $(1,60)$.

\section{Reconstruction of library functions}

Against excessive call library function in the algorithm problem, the reconstruction of library function ways to optimize the solution: the library function call simple, the macro definition; for the complex library function calls, look-up table method instead of. So on the one hand, reduce the library function call overhead, on the other hand, reduces the computational complexity of some library functions, so as to further improve the performance of the algorithm.

According to the algorithm in exp (x) function invocation context, analysis of the exp (x) parameter range function for $(-5,0)$. Because the interval contains infinite values, to ensure accuracy of the circumstances, the need for precision reasonable division of lookup table: All parameters of $1,0.1,0.01$. specific for each partition and memory overhead, performance, accuracy effect as shown in table 1 . In the premise of precisely $100 \%$, here is a selection of accelerated 0.1 relatively high precision production lookup table, the memory overhead is only 204bytes, less than $0.1 \%$ of total memory overhead.

Table 1 Precision performance test table

\begin{tabular}{|c|c|c|c|}
\hline Classification accuracy & The memory overhead /byte & Correct rate & Speedup \\
\hline
\end{tabular}




\begin{tabular}{|c|c|c|c|}
\hline 1 & 20 & $94 \%$ & 19 \\
\hline 0.1 & 204 & $100 \%$ & 4.45 \\
\hline 0.01 & 2040 & $100 \%$ & 0.51 \\
\hline
\end{tabular}

According to the analysis before, in the two call to BoxIntegral, two vertices of original algorithm will repeat visits two adjacent rectangular coincidence, caused by redundant memory a lot of access. This paper combines the two call to BoxIntegral, which is in the haarX function in the integral image ImageDate, the coincidence point only a visit. Through this operation, the original calculation of a Haar wavelet transform values need 8 times the memory access is reduced to 6, a decline of $25 \%$ memory access.

This paper is based on the work of the current mainstream SURF version of OpenSURF, version 310809, used in the graphic library is OpenCV, program analysis by using Intel's Profiling tool Vtine.

The experiment adopted a set of 48 image test set provided by KristianMikolajczy, and the BMP images with I turn yellow into standard 640 X 480 pixels, to facilitate other accelerated system test results. Performance tests were performed in 10 times, remove the fastest and most slowly case, take the arithmetic mean as the version of the operating time value.

\section{The experimental evaluation}

Test method for performance test set of 48 images is calculated 50 times, a total of 2400 images is calculated, can simulate the actual application scene in scale, more reasonably draw actual improved the speedup of the algorithm. Table 2 Comparison of the running time optimization of SURF and the original SURF.

The experiment adopted a set of 48 image test set provided by KristianMikolajczy[8], and the BMP images with I turn yellow into standard 640 X 480 pixels, to facilitate other accelerated system test results. Performance tests were performed in 10 times, remove the fastest and most slowly case, take the arithmetic mean as the version of the operating time value.

Table 2 The optimization performance compared with the original version version

\begin{tabular}{|c|c|c|c|c|}
\hline \multirow{2}{*}{\multicolumn{2}{|c|}{ Stage }} & \multicolumn{2}{|c|}{ Time consuming /ms } & \multirow{3}{*}{$\begin{array}{c}\text { Speedup } \\
1.0\end{array}$} \\
\hline & & \multirow{2}{*}{$\begin{array}{c}\text { The originl } \\
\text { version } \\
8426 \\
\end{array}$} & \multirow{2}{*}{$\begin{array}{l}\text { The optimizd version } \\
\qquad 8468\end{array}$} & \\
\hline Initialization & Image loading & & & \\
\hline \multirow{2}{*}{ Feature detection } & $\begin{array}{l}\text { Eigenvalue } \\
\text { calculation }\end{array}$ & 222669 & 31892 & 6.98 \\
\hline & $\begin{array}{l}\text { Feature point } \\
\text { selection }\end{array}$ & 18555 & 16753 & 1.11 \\
\hline $\begin{array}{c}\text { Characteristics of } \\
\text { phase }\end{array}$ & $\begin{array}{c}\text { The characteristic } \\
\text { direction calculation }\end{array}$ & 93348 & 81431 & 1.14 \\
\hline SURF & Total time & 748835 & 272203 & 2.75 \\
\hline
\end{tabular}

Overall, the optimization of SURF than the original SURF happy 2.75 times. At the same time, table 4 shows the various optimization strategies to the role of. As can be seen from the table:

Calculation of values in the feature detection, this paper determine the logic of the BoxIntegral function is reconstructed, using an extended array instead of all of the conditions, the optimal strategy to accelerate to 6.98 times.

Select the stage in the feature detection, while we do not directly aimed at the optimization 
strategy of the stage, but because the stage called simple library function min, Max, fRound have been optimized, reduce 1.11 times speedup.

Computation phases in the characteristic direction feature description, data locality optimization for haarX/haarY function in this paper, a decrease of $25 \%$ memory accesses, and reconfigurable logic judgment, achieved a speedup of 1.14 times.

The correctness of the test is divided into two parts: correctness testing feature detection stage correctness testing and feature description stage. Correctness testing feature detection stage is relatively primitive and feature point optimization version number is the same. Correctness test description stage is the product of the comparison of the original and optimized version of matching feature detection stage accuracy phase accuracy and feature description.

The reason for using the step accuracy is that this method can be matched to the feature point level of refinement and non image level, to maximize the detection optimization SURF algorithm is correct.

For the optimization of SURF and the original SURF, the total number of feature point test set of 48 pictures are 47454 , matching feature points are 47454 , the correct rate of two stages, so the optimization of SURF algorithm are $100 \%$, the correct rate of the whole algorithm is $100 \%$.

\section{Summary}

In this paper, analysis and performance evaluation of the mainstream view of the current image retrieval algorithm SURF to.Based on the conclusions of the analysis, to ensure $100 \%$ accuracy, the original SURF algorithm using the refactoring decision logic, data locality optimization, reduce the redundant memory access and look-up table method is improved and optimized, finally proposes an optimized version of the SURF algorithm, to improve the performance of $2.75 \mathrm{X}$.

\section{References}

[1] Lowe D.Object rec from local scale-invariant features[C]//n-ternation Conference on Computer Vision, Corfu,Greece,1999:1150-1157.

[2] LDG.Distintive image features from scale-invariant Keypoints[J].International Journal of Computer Vision,2004,60(2):404 - 407.

[3] B H,F B,,G L V.Interactive museum guide:Fast and robust recognition of museum objects[C]//The First International Workshop on Mobile Vision,2006.

[4]VM,GLV.Web-based3dreconstructionservice[J].MachineVisionand Applications,2006,17(6):411

[5] J Y,B S.Visualrank:Applying pagerank to large-scale image search[].IEEE Transactions on Pattern Analysis and Machine Intelligence,2008,30(11):1877 - 1890.

[6] Uijlings J R R,Smeulders A E M , Scha R J H, Real-time bag of words , approximately[C]//roceeding of the ACM International Conference on Image and Video Retrieval,2009:1 - 8.

[7] Heymann S, Muller K, Smolic A, et al. Sift implementation and optimization for general-purpose GPU[C]//Proc. WSCG’07,2007. 